\title{
Esophageal squamous cell cancer coincides with myelodysplastic syndrome/acute myelogenous leukemia: A case report and review of the literature
}

\author{
LINGLING LIU $^{1 *}$, XIAOYUN LIU ${ }^{2 *}$, YANLING SUN $^{1}$, YINZHU ZENG ${ }^{1}$, XIAOYUN TANG ${ }^{1}$, \\ XIAOXIA LI ${ }^{3}$, JIAJUN LIU ${ }^{1}$ and RUOZHI XIAO ${ }^{1}$
}

${ }^{1}$ Department of Hematology, The Third Affiliated Hospital of Sun Yat-Sen University and Sun Yat-Sen Institute of Hematology;
${ }^{2}$ Department of General Medicine, The Third Affiliated Hospital of Sun Yat-Sen University, Guangzhou, Guangdong 510630;
${ }^{3}$ Department of Molecular Pathology, KingMed Genome Diagnostic Laboratory, Guangzhou, Guangdong 510320, P.R. China

Received February 17, 2020; Accepted July 13, 2020

DOI: $10.3892 / \mathrm{ol} .2020 .12129$

\begin{abstract}
Esophageal squamous cell carcinoma (ESCC) is one of the most common malignant tumors, and has high incidence and mortality rates, worldwide. Myelodysplastic syndrome (MDS), a disorder of hematopoietic stem or progenitor cells, results in marrow failure, which increases the risk of acute myeloid leukemia (AML). Few studies had reported patients who have suffered from both ESCC and MDS/AML simultaneously. To identify possible potential associations between ESCC and MDS/AML, the present case report describes a patient with both types of these tumors at the same time. Following endoscopic biopsy, the patient was revealed to have moderately differentiated SCC. MDS with excess blasts was subsequently diagnosed following bone marrow aspiration. The results of next-generation sequencing revealed that TP53 and ROS1 were both found in ESCC and MDS/AML tumors. The patient refused therapeutic intervention and died within 20 days. The current report demonstrated that hematologic malignancies presenting alongside solid tumors should be considered clinically. In addition, the report indicated that bone marrow puncture should be performed in patients with solid tumors and abnormal blood test results. Next-generation sequencing may be a useful technique for the investigation of patients with two or more neoplasms. However, more research regarding the co-existence of solid tumors with hematological malignancy are required.
\end{abstract}

Correspondence to: Dr Ruozhi Xiao, Department of Hematology, The Third Affiliated Hospital of Sun Yat-Sen University and Sun Yat-Sen Institute of Hematology, 600 Tianhe Road, Guangzhou, Guangdong 510630, P.R. China

E-mail: liul135@mail.sysu.edu.cn

*Contributed equally

Keywords: esophageal squamous cell carcinoma, myelodysplastic syndrome, myeloid leukemia, next-generation gene sequencing

\section{Introduction}

Due to its aggressive nature and poor survival rates, esophageal cancer is the 6th leading cause of cancer-associated deaths and is the 8th most common cancer worldwide, according to GLOBOCAN 2008 (1). In Chinese men, esophageal cancer was the 5th most common cancer and the 4th leading cause of cancer-associated deaths in 2013 (2). Esophageal squamous cell carcinoma (ESCC) accounts for $88 \%$ of esophageal cancers (3). Myelodysplastic syndrome (MDS) is a disorder that is found in hematopoietic stem or progenitor cells, which is characterized by abnormal differentiation, dysplasia and peripheral blood cytopenias (4). Approximately $30 \%$ of patients with MDS subsequently develop acute myeloid leukemia (AML) (5). However, the underlying molecular mechanism of this process is unknown (5). MDS occurs more frequently in older patients ( $\geq 60$ years of age) and individuals who have been exposed to prior chemotherapy or radiation therapy, and has an overall survival (OS) time ranging from several months to 10 years (6). The prevalence of multiple primary malignant neoplasms (MPMN) has been demonstrated to range from 0.73 to $11.7 \%$ worldwide (7). The majority of synchronous malignancies are solid tumors; however, cases of primary MDS/AML occurring alongside solid tumors have rarely been reported. The current case report describes a patient with ESCC and MDS that rapidly progressed to AML. Next-generation gene sequencing was also performed to elucidate the associations between these types of cancer.

\section{Case report}

Clinical experiments. Bone marrow smear and cytochemical staining analysis were performed according to routine protocols by the clinical laboratory of the Department of Hematology of The Third Affiliated Hospital of Sun Yat-sen University (Guangzhou, China). The Wright-Giemsa stain was applied onto the whole smear for $10 \mathrm{sec}$ at room temperature, then the same amount (0.5-0.8 ml) of phosphoric acid buffer was added and mixed with the dye solution at room temperature for $25 \mathrm{~min}$. Subsequently, the smear was washed with distilled water and the slides were air-dried. 
Histopathology and hematoxylin and eosin (HE) staining analysis were performed by the Department of Pathology of The Third Affiliated Hospital of Sun Yat-sen University. HE staining was performed according to routine protocols. Briefly, after deparaffinization and rehydration, 5- $\mu \mathrm{m}$-thick sections were stained with hematoxylin solution for $5 \mathrm{~min}$ at room temperature, followed by 5 washes in $1 \%$ acid ethanol and then rinsed in distilled water. Subsequently, the sections were stained with eosin solution for 3 min at room temperature, followed by dehydration in a graded alcohol series and clearing in xylene. The mounted slides were then examined and photographed using a light microscope (Olympus BX43; Olympus Corporation; magnification, x100).

Flow cytometry and next-generation sequencing were performed by Kind Med Diagnostics Group Co., Ltd.

\section{Results}

A 51-year-old male patient had dysphagia, particularly with coarse food, for 10 days without the symptoms subsiding. After visiting a local hospital (Heyuan Peoples Hospital; Heyuan, Guangdong, China), the patient was diagnosed with ESCC after receiving biopsy using gastroscopy. He was subsequently admitted to The Third Affiliated Hospital of Sun Yat-Sen University (Guangdong, China) for further evaluation and treatment on 27th November 2018. He had no history of previous malignancies, exposure to ionizing radiation or chemotherapy. He also had no family history of cancer. He was a frequent smoker ( $>30$ cigarettes per day) and consumed $150 \mathrm{ml}$ alcohol per day for $\sim 30$ years. Enhanced computer tomography scans of the chest and upper abdomen indicated an esophageal neoplasm with multiple swollen lymph nodes within the mediastinum, and an unascertained nodule in the superior lobe of the left lung (Fig. 1A and B). The results of the endoscopy revealed a large semi-circular mass, covered with white moss in the lower esophagus (Fig. 1C). Following endoscopic biopsy, the mass was determined to be moderately differentiated squamous cell carcinoma (Fig. 1F). According to the 8th edition of the American Joint Committee on Cancer/ Union for International Cancer Control TNM staging system, the clinical stage of the patient was cT3N2M0, stage IVA (8). Routine blood analysis revealed elevated white blood cells $\left(8.99 \times 10^{9} / 1\right)$, and hemoglobin $(90 \mathrm{~g} / 1)$ and platelet $\left(25 \times 10^{9 / 1} / 1\right)$ counts. MDS with excess blasts was diagnosed according to the 2016 World Health Organization classification after bone marrow aspiration (9). The bone marrow smear revealed pathological hematopoiesis and $10.5 \%$ primordial immature granulocytes (Fig. 1D). Flow cytometry analysis of the bone marrow revealed $10 \%$ medullary primitive cells with abnormal immunophenotype, exhibiting $\mathrm{CD} 34^{+}, \mathrm{CD}_{117^{+}}, \mathrm{CD} 33^{+}$, human leukocyte antigen-DR phenotype ${ }^{+}, \mathrm{CD}^{-}{ }^{-}, \mathrm{CD} 64^{-}, \mathrm{CD} 56^{-}$, $\mathrm{CD}^{-}{ }^{-}, \mathrm{CD}^{+}$(few), CD5- and CD2- (Fig. 2). Cytogenetics analysis revealed that the patient exhibited a complex karyotype, with 44-48, xy, -4, 5, +6, del(7)(q21), del(9)(q21;q32), del(11)(q14), $+12,+12$, del (12)(p10), -15, +22, +mar, 1-5dmin (9) $46, x y(1)$, with one of these chromosome karyotypes shown in Fig. 3. After 10 days, the bone marrow smear demonstrated $25 \%$ primitive cell transformation to AML (Fig. 1E). The next-generation sequencing result revealed genetic mutations in TP53, ALK receptor tyrosine kinase (ALK), ROS proto-oncogene 1, receptor tyrosine kinase (ROS1), AT-rich interaction domain 1A (ARID1A), platelet-derived growth factor receptor $\beta$ (PDGFRB), cyclin-dependent kinase inhibitor 2A (CDKN2A) and neurofibromin 1 (NF1) in the ESCC samples, while mutations in TP53 and ROS1 were found in the bone marrow fluid (10). In ESCC samples, there were three point mutations of TP53, and the mutation frequencies were 6\% for TP53:NM_000546.5:c.764dupT(p. T256fs) exon 7, 4.5\% for TP53:NM_000546.5:c.711G $>$ A(p. M237I) exon 7 and 14.1\% for TP53:NM_000546.5:c.580C $>$ T (p.L194F) exon 6. In the bone marrow fluid, there were two mutations of TP53, and the mutation frequencies were $37.3 \%$ for TP53:NM_000546.5:c.764dupT(p.T256fs) exon 7 and 55.6\% for TP53:NM_000546.5:c.711G $>A(p . M 237 I)$ exon 7. The mutation frequency of ROS1:NM_002944.2:c.5842C $>$ T (p.R1948C) exon 36 was 41.4\% in ESCC samples and $45.2 \%$ in the bone marrow fluid (10). Due to the poor financial position of the patient, therapeutic intervention was refused and the patient died within 20 days.

\section{Discussion}

The etiology of ESCC is multi-factorial. It has been found that tobacco, alcohol, poverty, betel quid, pickled vegetables, hot foods, $\mathrm{X}$-rays, $\gamma$-radiation and achalasia increase the risk of developing ESCC (11). Genetic variants, such as frameshift or missense mutations, in TP53, RB transcriptional corepressor 1, CDKN2A, phosphatidylinositol-4,5-bisphosphate 3-kinase, catalytic subunit $\alpha$, notch receptor 1 , nuclear factor, erythroid 2 like 2, family with sequence similarity 135 member B, lysine methyltransferase 2A (KMT2A), ASH1 like histone lysine methyltransferase, KMT2C, SET domain containing $1 \mathrm{~B}$, histone lysine methyltransferase, CREB-binding protein and E1A binding protein p300, may serve important roles in the development of ESCC (12). The etiology and pathogenesis of MDS/AML has not been elucidated, as the primary cause of MDS is only established in 15\% of cases (13). Benzene exposure is one of the few well-known risk factors for myeloid malignancy (14). However, exposure to soot, creosote, inks, dyes, tanning solutions and coal dust may also serve a role in the etiology of myeloid malignancy (14). Du et al (15), found that MDS was significantly associated with smoking, but not with alcohol consumption. The patient in that study, was a frequent tobacco and alcohol consumer, exhibited various well-known cancer-associated genetic mutations and suffered from ESCC. Multiple primary malignant neoplasms (MPMNs) are rare malignant neoplasms that simultaneously or successively occur in one patient, as two or more primary malignancies (16). In general, MPMNs are more common as two solid tumors or as two hematological malignancies. Cases of MPMN that involve solid tumors and hematological malignancies are; however, rare (16). To the best of our knowledge, only one previous report has described a patient with AML with ESCC (17). Li et al (16), reported a case of colorectal cancer and multiple myeloma (MM) with chest wall involvement. In that case, MM was confirmed following two cycles of chemotherapy for colorectal cancer. The authors were therefore unable to conclude whether the two malignancies were associated. However, in the present case, ESSC and MDS/AML were confirmed almost at the same 


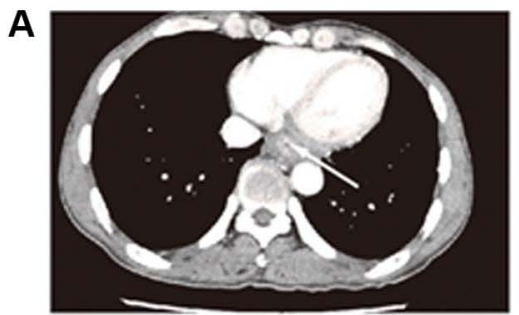

D

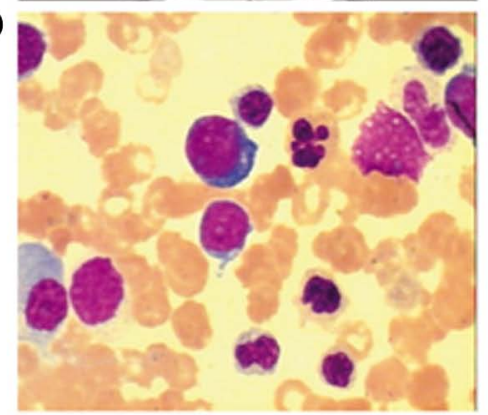

B

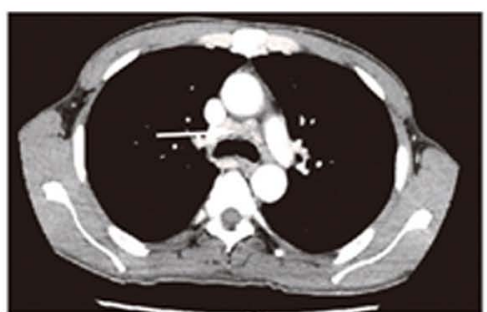

E

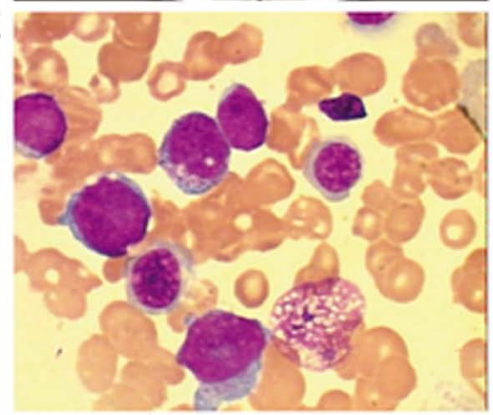

C

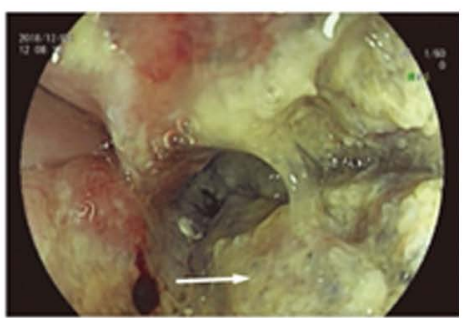

$\mathbf{F}$

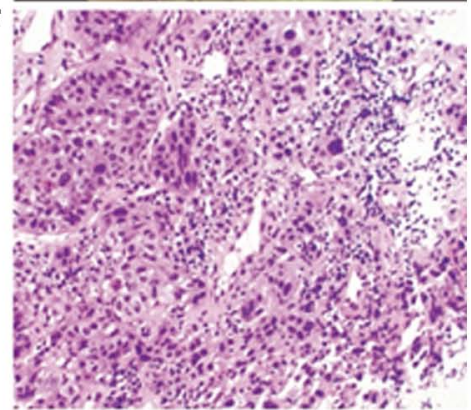

Figure 1. Clinical examinations of the patient. (A) Contrast-enhanced CT of the chest revealed lower esophageal strengthened mass. (B) Contrast CT of the chest revealed mediastinal enlargement of the lymph nodes. (C) Endoscopy showed a semi-circular giant mass covered with white moss. (D) Bone marrow smear revealed pathological hematopoiesis and $10.5 \%$ primordial immature granulocytes (magnification, $\mathrm{x} 1,000)$. (E) Bone marrow smear showed medullary primitive cells up to $25 \%$ after 10 days. (F) Biopsy of the esophageal using endoscopy showed moderately differentiated esophageal squamous cell carcinoma, using hematoxylin and eosin stain (magnification, x100). CT, computer tomography.

A

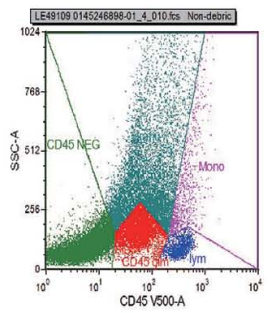

$\mathrm{C}$

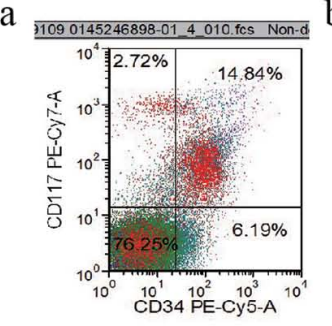

$\mathrm{b}$

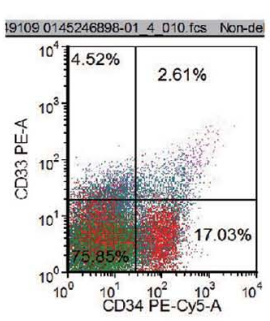

$\mathrm{D}$

a

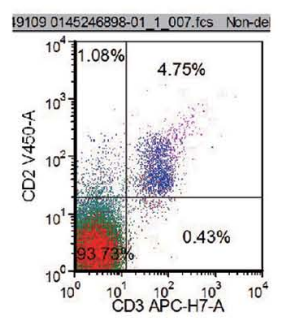

B
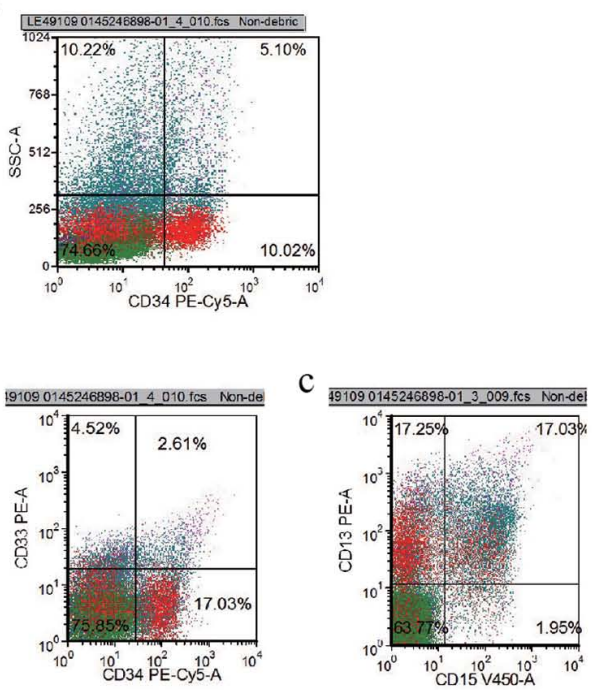

d

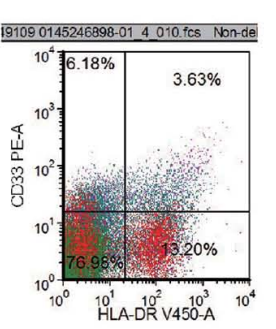

e

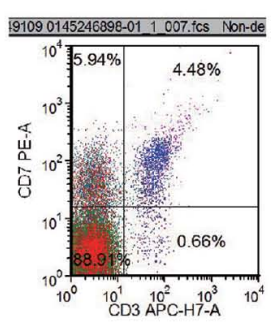

d

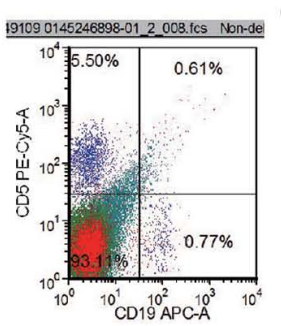

e

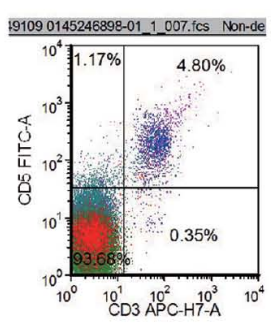

Figure 2. Flow cytometry assay revealed primordial immature granulocytes in the bone marrow of the patient. (A) $\mathrm{CD} 45^{+}$cells. (B) $\mathrm{CD} 34^{+}$cells. (C-a) $\mathrm{CD} 117^{+}$ $\mathrm{CD} 34^{+}$, (C-b) $\mathrm{CD}_{3}{ }^{+} \mathrm{CD} 34^{+}$, (C-c) $\mathrm{CD}_{3} 3^{+} \mathrm{CD} 15^{+}$, (C-d) human leukocyte antigen-DR phenotype ${ }^{+} \mathrm{CD} 33^{+}$and (C-e) $\mathrm{CD}^{+} \mathrm{CD}^{-}$cells. (D-a) $\mathrm{CD}^{-} \mathrm{CD}^{-}$, (D-b) CD14 CD64, (D-c) CD56 CD3*, (D-d) CD19- CD5 and (D-e) CD5 CD3 cells.

time. Unexplained thrombocytopenia required the patient in the present case report to undergo a bone marrow aspiration, which was important for the diagnosis of MDS/AML.
A karyotype with $>4$ aberrations, including translocations, inversions and copy number insertions or deletions, was found to be a significant adverse-risk abnormality in 




Figure 3. One of the chromosome karyotypes of the patient. 48, xy, $-4,-5,+6, \operatorname{del}(7)(\mathrm{q} 21), \operatorname{del}(9)(\mathrm{q} 21 ; \mathrm{q} 32), \operatorname{del}(11)(\mathrm{q} 14),+12,+12, \operatorname{del}(12)(\mathrm{p} 10),-15,+22,+\mathrm{mar}$, $2 \mathrm{dmin}$. The arrows indicate the abnormal chromosomes.

AML (18). The complex karyotype of the patient in the present case study containing $>4$ aberrations suggested a poor prognosis. Next-generation sequencing revealed that several gene variants existed in the bone marrow and ESCC of the patient. ARID1A, as a novel tumor suppressor, is essential for maintaining the frequency and function of hematopoietic stem cells, and was found to be associated with gynecological, liver, gastric and breast cancer, as well as leukemia (19). While NF1 mutations, including missense, frameshift and nonsense mutations, in patients with AML were indicative of an poor prognosis (20), they are also found in patients with ESCC (21). The PDGFRB gene, is located on chromosome 5q31-33 (22). Patients with lymphocytic leukemia and AML with PDGFRB fusions could be treated with tyrosine kinase inhibitors $(23,24)$. PDGFRB regulates the mRNA expression of transforming growth factor- $\alpha$ and serves a central role in esophageal carcinoma tumor invasion and metastasis (25). The next-generation sequencing results, from the patient in the present study, revealed that the genetic mutations in TP53 and ROS1 were found in both ESCC and MDS/AML. The ROS1 mutation frequency was $45.2 \%$ in MDS/AML and $41.4 \%$ in ESCC (10). The ROS1 gene belongs to the sevenless subfamily of tyrosine kinase insulin receptor genes (26). ROS1 protein expression in adult humans is highest in the kidney; however, it is also found in the cerebellum, peripheral neural tissue, stomach, small intestine, and colon, with lower expression levels in parathyroid, larynx, adrenal gland and skeletal muscle tissues (27). However, to the best of our knowledge, this is the first time that ROS1 was detected in ESCC in the patient, in the present case report. The ROS1 gene often forms fusion gene with other genes, such as ALK (26). The signaling pathways, such as Ras/Raf/MEK/ERK1/2 and JAK/STAT, activated by ROS1 fusions appear to primarily involve common growth and survival pathways $(26,28)$. The Food and Drug Administration approval of tyrosine kinase inhibitors for the treatment of ALK- and ROS1-rearranged non-small cell lung cancer led to the understanding that receptor tyrosine kinase fusions could be targets for the treatment of oncogenic driver alterations in a diverse group of solid malignancies, such as gastric cancer (29). However, the function of ROS1 mutations in ESCC of the present patient requires further investigation. The TP53 point mutations frequency was much lower in ESCC [6\% for NM_000546.5:c.764dupT(p.T256fs) exon 7 and 4.5\% for NM_000546.5:c.711G>A(p.M237I) exon 7] compared with that in MDS/AML [37.3\% for NM_000546.5:c.764dupT(p. T256fs) exon 7 and 55.6\% for NM_000546.5:c.711G>A(p. M237I) exon 7] in the patient in the present case study. There are two possible reasons that may explain this phenomenon: First, ESCC may have had fewer TP53 mutations; second, TP53 mutations in ESCC may have resulted from a small quantity of MDS/AML cell infiltration, which would also explain the lower detection rate of TP53 mutation frequency in ESCC. TP53, a tumor suppressor gene located on chromosome $17 \mathrm{p} 13$, serves crucial roles in tumor genesis (30) and is the most frequently mutated gene in human cancer, with a frequency of $\sim 50 \%$ in squamous cell skin carcinoma (31). In addition, TP53 has been identified in numerous different types of hematological malignancies, such as AML, MDS and acute lymphoblastic leukemia (31). The p53 mutation in codon 248 (p53R248W) was revealed to enhance leukemia-initiating cell self-renewal to promote leukemia development (32). Thus, genetic mutations in TP53 and ROS1 were the most important oncogenes that were identified in the patient, in the present case report. 
Variants in the TP53, ROS1, ALK, ARID1A, PDGFRB, CDKN2A and NF1 genes are important in patients with ESCC and MDS/AML as they have been associated with tumor formation, recurrence and metastasis. In the patient in the present case study, mutations in these genes might have been involved in the development of ESSC and MDS/AML. However, further investigation regarding how the gene mutations may promote the proliferation of tumor cells is required for confirmation. The presence of these gene variants, in addition to tobacco and alcohol consumption, may have resulted in the patient developing MPMN. The patient refused chemotherapy; therefore, the effect of therapeutic intervention was unknown, although there is no standard treatment regimen for MPMN and it is unknown which malignant neoplasm should be prioritized. Thus, further research is required to determine this.

In conclusion, the current case report describes an extremely rare case of ESCC presenting in conjunction with MDS/AML. The results indicated that for patients with solid tumors and unexplained blood changes, the presence of a hematological malignancy should be considered and bone marrow puncture should also be performed. The application of next-generation gene sequencing may also provide more information regarding gene mutations, which may also guide future treatment. The poor lifestyle of the patient in the current case study may have promoted the development of MPMN, which requires multiple gene mutations. However, the underlying molecular mechanism of this process requires further investigation.

\section{Acknowledgements}

Not applicable.

\section{Funding}

The present study was supported by the Fundamental Research Funds for the Central Universities (grant no. 20YKPY29) and the Science and Information Technology Bureau of Guangzhou Haizhu District (grant no. 2012-ZD-02).

\section{Availability of data and materials}

The data that support the findings of this study are available from reference (10) (dataset posted in May 2020).

\section{Authors' contributions}

RX supervised the writing of the manuscript and diagnosis and treatment of the patient. JL supervised the overall care of the patient. XYL wrote the manuscript. LL performed bone marrow aspiration and revised the manuscript. YS analyzed the data and edited the figures. XT helped revise the manuscript. XXL performed next-generation gene sequencing and acquired the data form Kind Med Diagnostics Group Co., Ltd. YZ performed the karyotype analysis. All authors approved the final version of the manuscript.

\section{Ethics approval and consent to participate}

The patients next of kin provided written informed consent.

\section{Patient consent for publication}

The patients next of kin provided consent for publication.

\section{Competing interests}

The authors declare that they have no competing interests.

\section{References}

1. Ferlay J, Shin HR, Bray F, Forman D, Mathers C and Parkin DM: Estimates of worldwide burden of cancer in 2008: GLOBOCAN 2008. Int J Cancer 127: 2893-2917, 2010

2. Chen W, Zheng R, Zhang S, Zeng H, Xia C, Zuo T, Yang Z, Zou $\mathrm{X}$ and He J: Cancer incidence and mortality in China, 2013. Cancer Lett 401: 63-71, 2010.

3. Arnold M, Soerjomataram I, Ferlay J and Forman D: Global incidence of oesophageal cancer by histological subtype in 2012 . Gut 64: 381-387, 2015.

4. Tefferi A and Vardiman JW: Myelodysplastic syndromes. N Engl J Med 361: 1872-1885, 2009.

5. da Silva-Coelho P, Kroeze LI, Yoshida K, Koorenhof-Scheele TN, Knops R, van de Locht LT, de Graaf AO, Massop M, Sandmann S, Dugas M, et al: Clonal evolution in myelodysplastic syndromes. Nat Commun 8: 15099, 2017.

6. Montalban-Bravo G and Garcia-Manero G: Myelodysplastic syndromes: 2018 update on diagnosis, risk-stratification and management. Am J Hematol 93: 129-147, 2018.

7. Demandante CG, Troyer DA and Miles TP: Multiple primary malignant neoplasms: Case report and a comprehensive review of the literature. Am J Clin Oncol 26: 79-83, 2003.

8. Rice TW, Ishwaran H, Ferguson MK, Blackstone EH and Goldstraw P: Cancer of the esophagus and esophagogastric junction: An eighth edition staging primer. J Thorac Oncol 12: 36-42, 2017.

9. Arber DA, Orazi A, Hasserjian R, Thiele J, Borowitz MJ, Le Beau MM, Bloomfield CD, Cazzola M and Vardiman JW: The 2016 revision to the World Health Organization classification of myeloid neoplasms and acute leukemia. Blood 127: 2391-2405, 2016

10. Liu L, Liu X, Sun Y, Zeng Y, Tang X, Li X, Liu J, Xiao R, et al: Esophageal squamous cell cancer coincidence with myelodysplastic syndrome/acute myelogenous leukemia. Figshare: doi. org/10.6084/m9.figshare.12349877.v1. Accessed May 21, 2020.

11. Abnet CC, Arnold M and Wei WQ: Epidemiology of esophageal squamous cell carcinoma. Gastroenterology 154: 360-373, 2018.

12. Song Y, Li L, Ou Y, Gao Z, Li E, Li X, Zhang W, Wang J, Xu L, Zhou Y, et al: Identification of genomic alterations in oesophageal squamous cell cancer. Nature 509: 91-95, 2014.

13. Adès L, Itzykson R and Fenaux P: Myelodysplastic syndromes. Lancet 383: 2239-2252, 2018.

14. Poynter JN, Richardson M, Roesler M, Blair CK, Hirsch B, Nguyen P, Cioc A, Cerhan JR and Warlick E: Chemical exposures and risk of acute myeloid leukemia and myelodysplastic syndromes in a population-based study. Int J Cancer 140: 23-33, 2017.

15. Du Y, Fryzek J, Sekeres MA and Taioli E: Smoking and alcohol intake as risk factors for myelodysplastic syndromes (MDS). Leuk Res 34: 1-5, 2010.

16. Li QL, Ma JA, Li HP, Huang RB, Hu CH, Liu XL, Gao YW, Feng GH and Wu F: Synchronous colorectal cancer and multiple myeloma with chest wall involvement: Is this a coincidence? Curr Probl Cancer 41: 413-418, 2017.

17. Hara M and Hoshijima T: Philadelphia chromosome (Ph1) positive acute myelomonocytic leukemia with esophageal cancer: A case report. Rinsho Ketsueki 33: 964-968, 1992 (In Japanese).

18. Stölzel F, Mohr B, Kramer M, Oelschlagel U, Bochtler T, Berdel WE, Kaufmann M, Baldus CD, Schafer-Eckart K, Stuhlmann R, et al: Karyotype complexity and prognosis in acute myeloid leukemia. Blood Cancer J 6: e386, 2016.

19. Han L, Madan V, Mayakonda A, Dakle P, Woon TW, Shyamsunder P, Nordin HBM, Cao Z, Sundaresan J, Lei I, et al: Chromatin remodeling mediated by ARID1A is indispensable for normal hematopoiesis in mice. Leukemia 33: 2291-2305, 2019.

20. Eisfeld AK, Kohlschmidt J, Mrozek K, Mims A, Walker CJ, Blachly JS, Nicolet D, Orwick S, Maharry SE, Carroll AJ, et al: NF1 mutations are recurrent in adult acute myeloid leukemia and confer poor outcome. Leukemia 32: 2536-2545, 2018. 
21. Hu N, Kadota M, Liu H, Abnet CC, Su H, Wu H, Freedman ND, Yang HH, Wang C, Yan C, et al: Genomic landscape of somatic alterations in esophageal squamous cell carcinoma and gastric cancer. Cancer Res 76: 1714-1723, 2016.

22. Golub TR, Barker GF, Lovett M and Gilliland DG: Fusion of PDGF receptor beta to a novel ets-like gene, tel, in chronic myelomonocytic leukemia with $\mathrm{t}(5 ; 12)$ chromosomal translocation. Cell 22: 307-316, 1994.

23. Heilmann AM, Schrock AB, He J, Nahas M, Curran K, Shukla N, Cramer S, Draper L, Verma A, Erlich R, et al: Novel PDGFRB fusions in childhood B- and T-acute lymphoblastic leukemia. Leukemia 31: 1989-1992, 2017.

24. Shimomura Y, Maruoka H and Ishikawa T: Marked response to imatinib mesylate in a patient with platelet-derived growth factor receptor beta-associated acute myeloid leukemia. Int J Hematol 105: 697-701, 2017.

25. Yoshida K, Kuniyasu H, Yasui W, Kitadai Y, Toge T and Tahara E: Expression of growth factors and their receptors in human esophageal carcinomas: Regulation of expression by epidermal growth factor and transforming growth factor alpha. J Cancer Res Clin Oncol 119: 401-407, 1993.

26. Uguen A and De Braekeleer M: ROS1 fusions in cancer: A review. Future Oncol 12: 1911-1928, 2016.
27. Rimkunas VM, Crosby KE, Li D, Hu Y, Kelly ME, Gu TL, Mack JS, Silver MR, Zhou X and Haack H: Analysis of receptor tyrosine kinase ROS1-positive tumors in non-small cell lung cancer: Identification of a FIG-ROS1 fusion. Clin Cancer Res 18: 4449-4457, 2012.

28. Roskoski R Jr: ROS1 protein-tyrosine kinase inhibitors in the treatment of ROS1 fusion protein-driven non-small cell lung cancers. Pharmacol Res 121: 202-212, 2017.

29. Drilon A, Ou SI, Cho BC, Kim DW, Lee J, Lin JJ, Zhu VW, Ahn MJ, Camidge DR, Nguyen J, et al: Repotrectinib (TPX-0005) is a next-generation ROS1/TRK/ALK inhibitor that potently inhibits ROS1/TRK/ALK solvent-front mutations. Cancer Discov 8: 1227-1236, 2018.

30. Isobe M, Emanuel BS, Givol D, Oren M and Croce CM: Localization of gene for human p53 tumour antigen to band 17p13. Nature 320: 84-85, 1986.

31. Preudhomme $C$ and Fenaux P: The clinical significance of mutations of the P53 tumour suppressor gene in haematological malignancies. Br J Haematol 98: 502-511, 1997.

32. Nabinger SC, Chen S, Gao R, Yao C, Kobayashi M, Vemula S, Fahey AC, Wang C, Daniels C, Boswell HS, et al: Mutant p53 enhances leukemia-initiating cell self-renewal to promote leukemia development. Leukemia 33: 1535-1539, 2019. 